\title{
P I 7- I 2. Immunogenicity comparison of dna vaccines encompassing HIV-I tat, rev, integrase (C-half), vif and nef genes derived from different clades
}

\author{
Y Wan*1, C Zhang ${ }^{2}, \mathrm{~J} \mathrm{Shi}^{3}, \mathrm{C}$ Qiu${ }^{4}, \mathrm{~S}$ Yuan$^{1}$ and J Xu${ }^{4}$
}

Address: ${ }^{1}$ Shanghai Public Health Clinical Center, Shanghai, PR China, ${ }^{2}$ Peking Union Medical College, Tisinghua University, Beijing, PR China, ${ }^{3}$ Institute of Molecular Biology, China Three Gorges University, Yichang, PR China and ${ }^{4}$ Institutes of Biomedical Sciences, Fudan University, Shanghai, PR China

* Corresponding author

from AIDS Vaccine 2009

Paris, France. 19-22 October 2009

Published: 22 October 2009

Retrovirology 2009, 6(Suppl 3):P294 doi:10.1 186/I742-4690-6-S3-P294

This abstract is available from: http://www.retrovirology.com/content/6/S3/P294

(c) 2009 Wan et al; licensee BioMed Central Ltd.

\section{Background}

B', B'/C and AE recombinant subtype of HIV-1 occupy the top three of the most prevalent HIV-1 clades in China. Thus, the preliminary object for HIV vaccine development in China is to obtain an efficacious vaccine targeting these clades. In order to assess their potencies to be used as $\mathrm{T}$ cell vaccine immunogen, we compared the immunogenicity of DNA vaccines containing tat, rev, integrase (C-half), vif and nef genes derived from the above three subtypes in mice model.

\section{Methods}

Polynecleotides encompassing tat, rev, integrase (C-half), vif and nef genes of HIV-1cn54(B'/C), HIV-1rl42(B') and HIV-1ae2f(AE) were synthesized respectively after codon optimization according to the preference of homo sapiens. Among these genes, tat, integrase(C-half) and nef were modified to ensure they were safe. The synthesized DNA fragments were transferred into plasmid vector to generated 3 DNA vaccines for vaccination of female Balb/ c mice in a DNA prime-DNA boost regimen. IFN- $\gamma$ Elispot assay was used to read out the T cell immunity.

\section{Results}

The general magnitude of T cell response elicited by pSVTRIVN(ae2f) (948 \pm 330 SFCs/106 splenocytes) was significantly higher than those of pSV-TRIVN(rl42) (195.1 \pm 44 SFCs/106 splenocytes) and pSV-TRIVN(cn54) (89.5 \pm 17 SFCs/106 splenocytes), $\mathrm{p}<0.01$. And most potent response was elicited by Integrase(C-half) and the second was Vif. While, rare response was induced by Tat, Rev and Nef.

\section{Conclusion}

Our data demonstrated that DNA vaccine derived from $\mathrm{HIV}-1 \mathrm{ae} 2 \mathrm{f}$ was most immunogenic among these three DNA vaccines. And in our vaccine formulation, the C-half of Integrase was the most immunogenic component. 